\title{
BMJ Open Acceptance and commitment therapy meets peer-support. Development of a supportive self-care intervention for patients with rare diseases: a multistage development process
}

\author{
Miriam K Depping (D), Natalie Uhlenbusch (D), Bernd Löwe
}

To cite: Depping MK, Uhlenbusch N, Löwe B. Acceptance and commitment therapy meets peer-support. Development of a supportive self-care intervention for patients with rare diseases: a multistage development process. BMJ Open

2021;11:e042856. doi:10.1136/ bmjopen-2020-042856

- Prepublication history and additional supplemental materials for this paper is available online. To view these files, please visit the journal online (http://dx.doi.org/10. 1136/bmjopen-2020-042856)

Received 22 July 2020 Revised 02 February 2021 Accepted 05 February 2021

Check for updates

(C) Author(s) (or their employer(s)) 2021. Re-use permitted under CC BY-NC. No commercial re-use. See rights and permissions. Published by BMJ.

Department of Psychosomatic Medicine and Psychotherapy, University Medical Center Hamburg-Eppendorf, Hamburg, Germany

Correspondence to

Dr Miriam K Depping;

m.depping.ext@uke.de

\section{ABSTRACT}

Objective We aimed at developing a patient-centred selfhelp programme, tailored to the needs of patients with rare chronic diseases.

Design Multistage, multimethod development process including a survey with validated self-report scales and open-ended questions (phase 1) and focus groups (phase 2) for needs assessment and, consolidating the first phases and the literature, the intervention development (phase 3).

Setting Phase 1: nationwide online survey in Germany, phase 2: four separate and diagnostically homogeneous focus groups. The focus groups took place at a university medical centre in Germany.

Participants Target group were patients with rare diseases that occur at a prevalence $<1: 2000$. Phase 1 : $\mathrm{n}=300$ participants with different rare diseases. Phase 2: Individuals with neurofibromatosis type $1(n=4)$, primary sclerosing cholangitis $(n=5)$, pulmonary arterial hypertension $(n=4)$ and Marfan syndrome $(n=5)$.

Results The central results of phases 1 and 2 were requests for more information about the disease and contact with other affected persons. Patients also expressed support needs in dealing with difficult emotions and identified acceptance of the disease as a turning point for quality of life. In phase 3 , we identified acceptance and commitment therapy (ACT) as a suitable therapeutic approach and determined details about implementation and execution of self-management programmes. A 6-week self-help concept led by peer counsellors was developed, which includes disease-specific information and ACTbased exercises.

Conclusion Based on a multistage needs assessment, we developed a peer-guided self-help intervention for patients with rare chronic diseases. Combining self-management, peer-counselling and ACT may help living with a rare condition. Further research needs to test the programme's efficacy.

Trial registration number ISRCTN13738704.

\section{INTRODUCTION}

There are around 7000 rare diseases worldwide (prevalence <1:2000), which differ greatly in their symptoms and course. ${ }^{1}$ In
Strengths and limitations of this study

- This is the first approach to developing an intervention tailored to the shared needs of patients with rare diseases.

- The intervention aims at providing psychosocial support for patients with different rare diseases concurrently who usually present as one of a kind in primary care.

- The development process includes assessing psychosocial care needs of this group of patients and is informed by proven frameworks and therapeutic techniques from other contexts.

- Needs assessment comprises quantitative survey data from a large group of heterogeneous patients as well as in-depth insight from focus groups with small homogeneous samples, thereby complementing methodological strengths and limitations.

- The current work describes the development process only, future research needs to test the intervention's efficacy and feasibility.

the face of finite healthcare resources, identifying commonalities in the experience and care needs can help to develop services for the entire group of patients that can improve their quality of life. Despite the heterogeneity, shared burdens can be assumed such as loss of control, reduced quality of life, lack of contact with other patients and often inadequate medical care. ${ }^{2}$

Chronic conditions cause psychosocial stress. ${ }^{3}$ Self-management approaches have been developed to help patients build skills in managing the challenges of living with a chronic condition. ${ }^{4}$ Strengthening patients in dealing with the psychological impact of living with a chronic condition can help improve quality of live. ${ }^{4}$ In individuals affected by common chronic conditions, depressive symptoms have been associated with a poorer somatic outcome, longer hospital stays and 
increased morbidity and mortality. ${ }^{5}$ Consideration of the mental health of physically ill patients is therefore a possible starting point for interventions that improve the quality of life. As most rare diseases cannot be cured, ${ }^{6}$ psychological intervention can support the management of the disease and thus change the overall quality of life. This creates scope for action for practitioners and patients.

It is likely that the experiences of patients with rare disease resemble those of patients affected by more common chronic conditions. In addition to the burden of chronic disease, patients with rare chronic disease may face additional stress. One source of additional stress may be uncertainty about their condition since rare conditions are often less well understood. Another source of additional stress may be geographical distribution. Since only few patients share a rare diagnosis, patients often live far apart which creates a barrier to psychosocial peersupport, self-help offers and adequate treatment.

We assume that individuals affected by rare chronic diseases are subject to specific burden due to the low prevalence of each disease by definition. A study commissioned by the German Federal Ministry of Health, in which both practitioners and representatives of patient organisations were interviewed, concluded that the burdens specific to rare diseases lead to a significant impairment of quality of life. ${ }^{7}$ The authors reported that there is a severe shortage of care services, especially primary care in the specialist area, which results in long diagnostic paths. A lack of information in many areas is also mentioned. In particular, the study identifies gaps in the care system, but does not ask about the everyday experience of those affected. Affected persons are also not directly interviewed in this study. However, the special challenge and necessity of interviewing the target group is emphasised.

Even more than the diagnosis itself, the perception of the disease and how the disease is dealt with has an impact on the quality of life of patients with rare conditions. ${ }^{8}$ A review of previous qualitative studies in the field of rare diseases describes that those affected report a lack of information and the need for more control. ${ }^{2}$ The authors show that emotional reactions, evaluations of the situation and coping strategies in dealing with the disease influence the quality of life. The authors also emphasise that studies on individual rare diseases have so far predominated and that there is still little knowledge about the shared experiences of those affected. Thus, it is still not well understood what it means to live with a rare disease. Therefore, additional studies are needed to inform the development of a novel intervention for patients with different rare diseases.

The aim of the research project was to narrow the gap in care for patients with rare diseases by developing of a self-help intervention tailored to the patients' needs. In this manuscript, we describe the systematic development of a short intervention to improve disease management in patients with rare diseases. Findings from a survey among patients with rare diseases and focus groups intending to inform the process are presented and discussed with respect to the content of the intervention.

\section{Multistage development process}

The first phase in the intervention planning involved assessing patients' needs across diagnoses. In the second phase, we assessed burden, needs and coping strategies qualitatively in four specific rare diseases. The third phase aimed at developing the intervention based on the results of the pre-studies and targeted literature search addressing open questions for intervention development. Figure 1 shows our multistage development process.

\section{Patient and public involvement}

This study is part of the research project 'patients for patients' aiming to develop and evaluate a disease management programme for patients with rare chronic diseases. We closely cooperate with a non-profit alliance (ACHSE e.V.) functioning as umbrella organisation for patient associations for rare diseases. ACHSE e.V. consulted the development, planning and implementation of the project by representing patients' interests. Further, we closely cooperate with patient associations directly who supported our recruitment process and help disseminate our results on their websites and in the associations' patient magazines.

\section{Phase 1: survey for cross-disease quantitative and qualitative needs assessment \\ Aims}

Which specific care needs can be addressed in order to contribute to the quality of life of patients despite chronic illness? Since hardly any data are available on the need for care of patients with different rare chronic diseases, we aimed at assessing the shared experiences of this patient group. Therefore, we investigated problems and support needs in patients with different rare chronic diseases.

\section{Methods}

In a cross-sectional online survey, we investigated the psychological burden and care needs in different areas of patients' lives. We included adult patients with different rare chronic diseases. We used quantitative measures to assess depression Patient Health Questionnaire (PHQ$9^{9-11}$ ) and anxiety Generalizied Anxiety Disorder Scale-7 $\left(\right.$ GAD- $\left.7^{12}{ }^{13}\right)$ as well as different psychosocial and diseaserelated characteristics. We further assessed problems and support wishes qualitatively with open questions. Ethics approval was given by the independent ethics committee of the Hamburg Medical Chamber (Ärztekammer Hamburg) on 2 February 2016 (reference number PV5088). A detailed description of the methods and results is described elsewhere, ${ }^{14}{ }^{15}$ please see online supplemental files for a complete list of survey instruments (online supplemental file 1 and patient flow online supplemental file 2). 


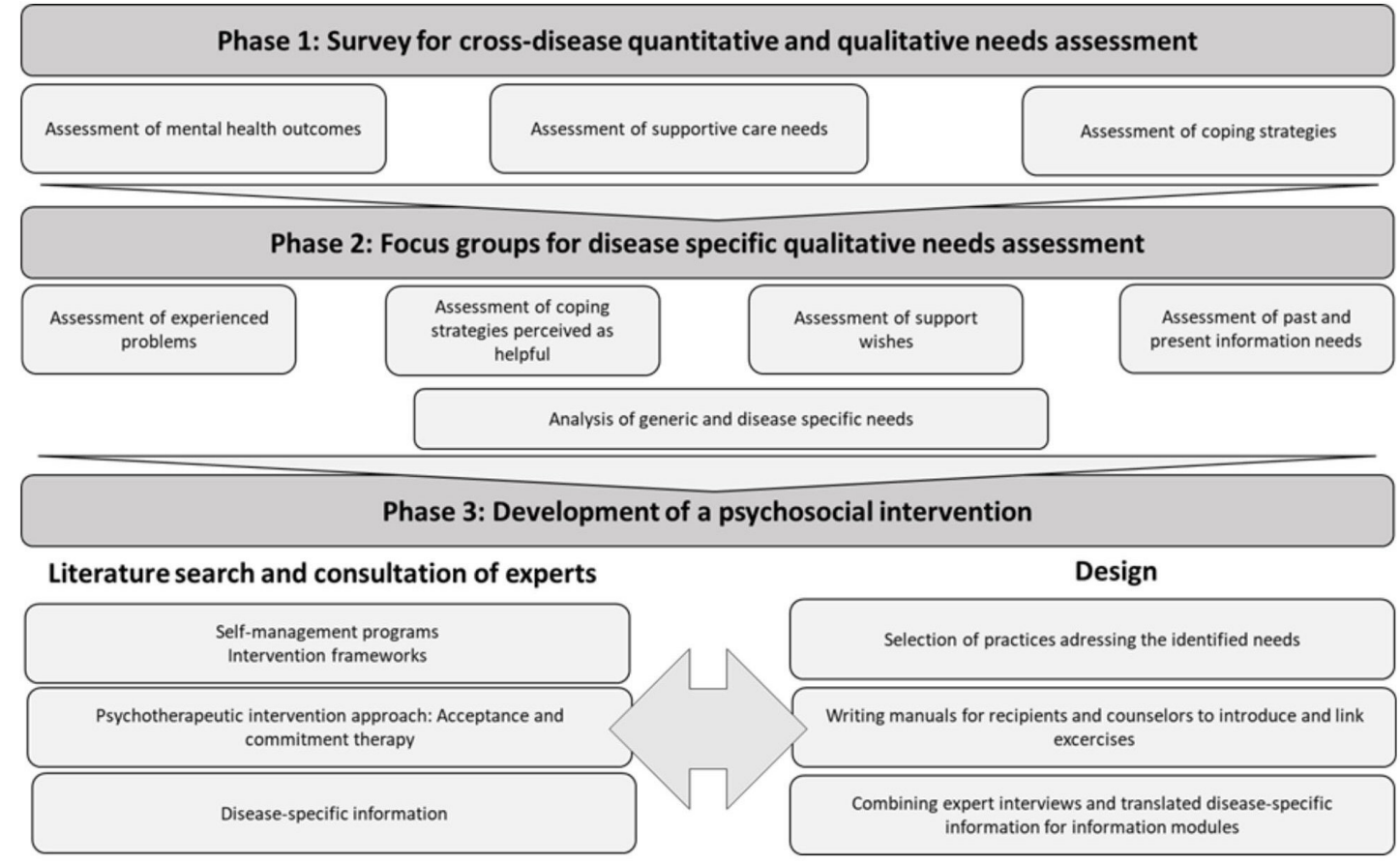

Figure 1 Development process.

\section{Results}

The study, which involved 300 patients with 79 different rare diseases, showed that patients with rare diseases are significantly more affected by depression and anxiety symptoms than the general population. ${ }^{14}$ Forty-two per cent of patients reported a clinically relevant depression burden with moderate to severe symptoms. For an anxiety disorder, this proportion was around $23 \%$. The study further revealed that psychosocial aspects contributed better to explained variation in depression and anxiety compared with disease-related variables. In particular, cognitive appraisal processes seem to play a role in the development of depression. A detailed description of the results on depression and anxiety is published elsewhere. $^{14}$

Our study further showed that patients with rare chronic diseases want more support in various areas of life than they currently experience and also clearly in comparison to a comparative sample of patients with cancer. ${ }^{15}$ The need refers for example to psychological support or assistance in everyday life. Moreover, patients stated the need for more information about the conditions. With regard to social support, a majority of the participants stated that they regularly had relieving conversations, in most cases with family members, partners and friends. Nevertheless, $59.9 \%$ of participants reported that they did not feel sufficiently supported socially.

\section{Conclusions}

The study results show high psychosocial burden on the patients as well as an increased and currently not sufficiently fulfilled need for support in different life areas including social support and information needs. We would like to stress five conclusions drawn from these results to inform the development of an intervention. First, the high psychopathological burden underlines the need for psychosocial support interventions. A second conclusion drawn from this study is that due to shared burden and experiences a generic intervention may be developed targeting patients with heterogeneous diagnoses. This is underlined by the fact that diagnosis-related variables did not contribute to explained variation in psychopathology, but psychosocial variables such as cognitive appraisal of the disease did. Thirdly, we conclude that targeting the perception of and attitudes towards a condition in the intervention can improve quality of life. These conclusions are further supported by the arguments of von der Lippe et $a l^{2}{ }^{2}$ A fourth conclusion pertains to the social context: an intervention may target the social relatedness of recipients in order to improve the perceived social support. In this regard, social support has been identified as an important protective factor against psychopathological symptoms in numerous studies with chronically physically ill patients. ${ }^{16}$

And finally, we conclude that a combination of generic and disease-specific elements seems most appropriate for the intervention. We assume that a generic intervention could help develop constructive attitudes towards the condition and fosters perceived social support. In contrast, the need for information can be best addressed specific to conditions.

\section{Phase 2: focus groups for disease-specific qualitative needs assessment \\ Aims}

Based on the assumption that disease-specific elements may be adequate, we chose to focus on four conditions which were all disabling yet hetergeneous. In order to 
further explore the burden of these diseases as well as support needs and what patients perceived as helpful in dealing with the diseases, we ran focus groups with patients affected by these four different conditions. The aim of the qualitative study was to assess diagnosis-specific as well as shared problems and psychosocial support needs. This study further guided the selection of the modules and development of disease-specific information modules.

\section{Methods}

In four diagnostically homogeneous focus groups, we asked patients with neurofibromatosis type 1, primary sclerosing cholangitis, pulmonary arterial hypertension and Marfan syndrome about problems as well as coping strategies with their rare disease. We chose conditions being treated at the University Medical Center HamburgEppendorf to facilitate recruiting in the face of low prevalence. We recruited adult patients with one of the four diagnoses via specialised outpatient clinics at the University Medical Center Hamburg-Eppendorf as well as patient organisations.

In all four focus groups, we used the same semistructured interview guide containing the following questions: 'Which aspects of the disease do you perceive as particularly burdensome? In what way does the disease impede your daily life? Which consequences does the disease have on your social life, your work or other areas of your life? What helps you deal with the burden of your rare chronic disease? What coping attempts have you already tried? What has helped and what has been less helpful? What else would you wish for?' (see online supplemental file 3 for a translation of the interview guide). The discussions were recorded, transcribed verbally and analysed with qualitative content analysis. ${ }^{17}$ We inductively derived categories regarding named coping strategies and support wishes from all four transcripts. To this end, two team members (NU, Anne-Marie Waßmuth (AW)) inductively and independently built categories according to the study aim based on reoccurring themes in all four pseudonymised transcripts. The resulting category system was discussed and revised several times with a third researcher (MKD). We identified superordinate themes and built main categories. Two independent team members deductively coded the four transcripts using the final category system. Two researchers (Laura Holz (LH), Johanna Dreyer (JD)) who participated neither in the focus groups nor in the development of the coding scheme, then independently coded the four transcripts using the final category system.

\section{Results}

We interviewed 18 patients with neurofibromatosis type $1(n=4)$, primary sclerosing cholangitis $(n=5)$, pulmonary arterial hypertension $(\mathrm{n}=4)$ or Marfan syndrome $(n=5)$ in four diagnostically homogeneous focus groups. Regarding problems associated with the diseases, we identified five main categories: medical problems, psychological burden, problems with the healthcare system, constraints and interpersonal problems. While medical problems differed widely between the diagnostic groups, patients with the different conditions independently reported many common problems including psychological burden, constraints in professional, personal and daily life, stigmatisation and others lacking understanding. A detailed description of these results is published elsewhere. ${ }^{18}$

Among aspects that help coping with the conditions, we identified five domains: social support, health services and treatments, profession and finances, leisure activities and psychological coping. Patients named aiding coping strategies they were able to carry out themselves (ie, adjusting leisure activities to new needs) or pertained to their evaluation of the situation (ie, acceptance of disease). Further aspects pertained to the system the patient is situated in (ie, social support) including healthcare services (ie, competent healthcare professionals, access to adequate treatment). An overview over all identified aspects is shown in table 1. Table 2 displays support wishes.

Numbers represent the total number of mentions and are displayed for each diagnosis individually as well as for the total number of mentions across all focus groups. The column $\mathrm{N}$ diag. represents the number of diagnoses, in which patients mentioned the respective category, with four representing all groups included in the study. Grey cells represent categories, which were mentioned at least once in a diagnostic group.

\section{Strategies and resources perceived as helpful}

Participants of the study were asked what has helped them coping with their disease in the past. Patients named assistive aspects from five areas: social support, health services and treatments, leisure, work and finance, psychological coping. Table 1 shows an overview of all categories revealed by qualitative content analysis.

\section{Social support}

Social support was reported as helpful, both by significant others as well as other patients affected by the disease.

So I can point out this DCCV [patient organization] again. They're doing a doctor-patient seminar. There's a federal meeting once a year, right? And then you get together with a lot of people and there are also a lot of transplanted and with them you can also have a little exchange. So wonderful evenings.

\section{Healthcare services and treatment}

Patients named access to specific treatments, information and aspects of patient-physician communication to be helpful.

...I am a strong person and nevertheless at first, I cried and it was so good that I here [in the hospital], the doctor has taken me in the arm, then came some sort of pastoral care. (...) so I practically arrived in the ambulance and somebody talked to me right away.-PAH 
Table 1 Results: what is perceived as helpful

\begin{tabular}{|c|c|c|c|c|c|c|}
\hline & NF & PSC & РAH & MFS & N Total & $\mathbf{N}$ diag. \\
\hline \multicolumn{7}{|l|}{ Social support } \\
\hline Social/emotional/ psychological support & 3 & 4 & 7 & 5 & 19 & 4 \\
\hline \multicolumn{7}{|l|}{ Healthcare services and treatment } \\
\hline Good patient-physicians relationship & 3 & & 2 & 2 & 7 & 3 \\
\hline Reachability of physicians & & 2 & & & 2 & 1 \\
\hline Good exchange between clinics & & & 1 & & 1 & 1 \\
\hline Surgeries & 3 & & 1 & & 4 & 2 \\
\hline Medication & 1 & 4 & 3 & & 8 & 3 \\
\hline Psychotherapy & 5 & & & 2 & 7 & 2 \\
\hline Consideration of patients' needs in professional life & 2 & 4 & 1 & 2 & 9 & 4 \\
\hline Financial security & & 1 & 1 & 1 & 3 & 3 \\
\hline \multicolumn{7}{|l|}{ Leisure activities } \\
\hline Massages & 1 & & & & 1 & 1 \\
\hline Positive activities & 4 & 3 & 1 & 4 & 12 & 4 \\
\hline Sports/exercise & 1 & 2 & 4 & 2 & 9 & 4 \\
\hline Relaxation/meditation & 3 & & & & 3 & 1 \\
\hline \multicolumn{7}{|l|}{ Psychological coping } \\
\hline Constructive attitudes & 3 & 9 & 4 & 10 & 26 & 4 \\
\hline Knowledge about the disease & & 5 & & 1 & 6 & 2 \\
\hline Spirituality & & & 1 & & 1 & 1 \\
\hline Structure in daily life & 1 & & & & 1 & 1 \\
\hline
\end{tabular}

MFS, Marfan syndrome; NF, neurofibromatosis; PAH, pulmonary arterial hypertension; PSC, primary sclerosing cholangitis.

\section{Work and finances}

Consideration of patients' needs and flexibility were named in all groups.

And then, with a new boss, I managed that I no longer have all shifts, because I noticed that sleeping doesn't work out well at all, even though I only work 20 hours, and I put my studies on hold so that I can still finish them... -MFS

\section{Leisure activities}

Patients in all groups named leisure activities as a form of self care. ...what really helped me back then, that I actually remained active and sporty (...). That helped me to survive these years, so to speak.- $\mathrm{PAH}$

\section{Psychological coping}

Acceptance, constructive attitudes and adaptation of a patients' lifestyle to the disease were reported as helpful in all focus groups.

And to have this very goal in mind, because now I can shape my profession, my path, my goal, my future self. This has given me a lot of energy to control my illness. Somehow already a lot. For example the cortisone therapy at the beginning was totally great, 
Table 2 Results: support wishes

\begin{tabular}{|c|c|c|c|c|c|c|}
\hline & NF & PSC & РAH & MFS & $\mathrm{N}$ total & $\mathrm{N}$ diag. \\
\hline \multicolumn{7}{|l|}{ Somatic care } \\
\hline Better professional competence & 10 & 4 & 2 & 3 & 19 & 4 \\
\hline Better patient-physician interaction & 7 & 5 & 1 & 6 & 19 & 4 \\
\hline Better/earlier diagnosis of the disease & 3 & & 3 & 2 & 8 & 3 \\
\hline More involvement in the treatment & 1 & 1 & & 2 & 4 & 3 \\
\hline More support from cost units & 7 & 1 & 4 & & 12 & 3 \\
\hline \multicolumn{7}{|l|}{ Psychological healthcare } \\
\hline Facilitated access to psychotherapy & 5 & & & 1 & 6 & 2 \\
\hline More psychological support in the treatment process & & 2 & & 6 & 8 & 2 \\
\hline \multicolumn{7}{|l|}{ Social support } \\
\hline More social/emotional/ psychological support & 2 & & 7 & 1 & 10 & 3 \\
\hline Exchange with other patients/ self-help groups & & & 1 & & 1 & 1 \\
\hline \multicolumn{7}{|l|}{ Personal resources } \\
\hline More self-confidence & 2 & & & 2 & 4 & 2 \\
\hline \multicolumn{7}{|c|}{ Better information and easier availability of information... } \\
\hline ...through more research & 1 & & & & 1 & 1 \\
\hline ...through more public awareness & & & 2 & 2 & 4 & 2 \\
\hline Information for patients & 1 & 8 & 4 & 2 & 15 & 4 \\
\hline Information for relatives & 2 & 1 & & & 3 & 2 \\
\hline \multicolumn{7}{|l|}{ Support for relatives } \\
\hline Support for relatives & 2 & & & 1 & 3 & 2 \\
\hline \multicolumn{7}{|l|}{ Reduction of somatic complaints and more functionality } \\
\hline Freedom from symptoms / more functionality & 2 & & 10 & 1 & 13 & 3 \\
\hline \multicolumn{7}{|l|}{ Assistance with formalities } \\
\hline Legal assistance & 1 & & 1 & & 2 & 2 \\
\hline Support with everyday formalities & 1 & & 2 & 1 & 4 & 3 \\
\hline
\end{tabular}

MFS, Marfan syndrome; NF, neurofibromatosis; PAH, pulmonary arterial hypertension; PSC, primary sclerosing cholangitis.

because I could say myself, now five up, now five down and I set the pace myself. That did me a lot of good.-PSC

\section{Support wishes}

Participants of the study were asked what type of support they wish for to help them cope with their disease in the future. Patients named assistive aspects from five areas: somatic care, psychological care, social support, information needs, support for relatives, reduction of somatic complaints and more functionality, assistance with formalities. Table 2 shows an overview of all categories revealed by qualitative content analysis.

Numbers represent the total number of mentions and are displayed for each diagnosis individually as well as for the total number of mentions across all focus groups. The column $\mathrm{N}$ diag. represents the number of diagnoses, in which patients mentioned the respective category, with 4 representing all groups included in the study. Grey cells represent categories, which were mentioned at least once in a diagnostic group.

\section{Conclusion}

With regard to problems as well as coping strategies, patients with different rare conditions named shared aspects in different domains. Medical problems were rather disease-specific, while there were many shared aspects among psychosocial aspects. With regard to support needs, patients also reported similar experiences across different conditions. At the same time, participants in all four focus groups wished for more information on their specific condition, replicating the finding from the survey-based assessment. This again highlights that combining disease-specific information modules with generic psychosocial elements may be most adequate for the planned intervention.

Regarding what helps to live with a rare chronic disease, patients named aspects from different domains: social support, health services and treatments, leisure, work and 
finance, psychological coping. The aspects mentioned included coping strategies that patients could implement themselves (eg, adaptation of leisure activities to new needs), while other aspects related to external support (eg, social support, competent medical staff). This underlines our conclusions from the survey-based needs assessment, that both addressing attitudes towards the diseases and the need for social support may have the potential to improve patients' quality of life. With regard to the attitudes towards the disease, patients across different conditions stated, that acceptance of the disease was a central process in adjusting to the disease and improving quality of life.

Some of the problems and support wishes patients named may be universal experiences of patients affected by chronic conditions, while other aspects could be specific to rare diseases. In conclusion, we decided to address aspects related to the rarity of the conditions in interventions, such as a lack of contact to peers with the same disease.

\section{Phase 3: development of a psychosocial intervention Aims}

Based on the assessment in the first two phases, we identified acceptance and commitment therapy (ACT) as a therapeutic approach in addressing the needs. ACT aims at supporting meaningful activity despite distress. ${ }^{19} 20$ To that end, processes of ACT include acceptance (ie, the willingness to experience distressing events without attempts to control them), and values-based action (ie, engaging in actions aligning with personally meaningful goals). ${ }^{1920}$ On the one hand this approach may promote the 'acceptance' patients described as central to disease adjustment in our focus groups; On the other hand this approach has been previously argued to suit the needs of patients affected by chronic conditions. ${ }^{21}$ To inform the development process, we reviewed literature on supportive care for patients with more common chronic conditions in self-management programmes. Further, we aimed at gathering information on how to best design a self-management intervention, that helps fostering constructive attitudes towards a disease (such as acceptance) and can be implemented transdiagnostically. We searched for details about implementation and execution of self-management programmes. Synthesising past and the presented research findings, we developed an intervention that facilitates peer-contact, provides information on the conditions and includes generic therapeutic modules fostering acceptance and commitment to meaningful goals.

\section{Methods}

In phase 3, we discussed the results of the first three phases in the core research team (MKD, NU and BL), with a group of clinicians and researchers in a research colloquium, with a group of experts in ACT for chronic conditions. In the development process, we built on the proven framework of self-management programmes and targeted the content to the needs of patients with rare chronic conditions. We handsearched for literature detailing delivery of self-management and ACT in general and for chronic conditions specifically. In order to address the need for social support and contact to peers with the same disease, we designed a programme, which can be delivered by a peer-counsellor. We developed a self-help manual including disease-specific as well as generic elements with information and exercises. We further developed consultation guidelines and a training concept for peer counsellors. The programme was designed for the same four rare diseases we included in the focus group study. Finally, we presented the concept and material of the intervention to a patient representative working for the German umbrella organisation of all rare disease patient organisations ACHSE e.V. who provided feedback on the intervention. When training the peer-counsellors we noted all feedback to any material, wording or exercise in the intervention and altered it in accordance with the patients' preference.

\section{Results}

Self-management programmes

For chronic conditions, self-management interventions have been implemented to complement primary care. ${ }^{4}$ These interventions aim at maximising patients' control over their health and thereby subjective quality of life. The interventions can focus on different contents including information on conditions, symptom management, psychological consequences and life style and combinations of them in multi-component programmes. ${ }^{22}$ Approaches targeting psychological consequences, life style, social support and communication are usually generic for different chronic conditions while components regarding information, medication and symptom management are disease-specific. ${ }^{22}$ The interventions are usually brief. Exemplary, the 'Chronic Disease SelfManagement Programme' is a 7-week, small-group intervention attended by people with different chronic conditions. The programme is mostly delivered by peer instructors from a highly structured manual who receive training for the task. ${ }^{23} 24$ Self-management programmes provided by paraprofessionals or "lay counsellors" (ie, trained individuals who are not licensed mental health providers) have been shown as equally effective to those led by healthcare professionals. ${ }^{22}$ The benefit of peer support has been emphasised for hardly reached groups of patients. ${ }^{25}$ On the one hand, peer counsellors can effectively deliver the intervention. On the other hand, they share experiences with the participants and may also be able to satisfy the need for more social connection and support. Thereby peer-counselling may benefit both recipient and counsellor.

\section{ACT as therapeutic approach}

ACT is a treatment approach enabling a transdiagnostic application, ${ }^{26}$ therefore, it can be applied irrespective of specific psychopathology or diagnosis. This is 
especially appropriate for a self-administered programme designed for a diverse group of patients. Rather than addressing specific psychiatric conditions, we chose ACT as it addresses the shared experiences and circumstances of patients with chronic conditions. In particular, by addressing acceptance as well as value orientation and commitment, ACT may foster the experience of meaning in patients' every day life. A key process in ACT is psychological flexibility, which is considered a protective factor against psychopathology. Processes to increase psychological flexibility include, among other things, active and aware acceptance of events even though they are unpleasant (eg, experiencing symptoms caused by a chronic illness) as well as implementation of value-based behaviour. ${ }^{19} 27$ ACT as group and individual therapy has been applied in a wide range of chronic conditions such as diabetes, cardiac disease and multiple sclerosis ${ }^{21}$ and can improve a variety of outcomes including quality of life, depression and psychopathology. ${ }^{21} 28$ Acceptance of adverse experiences seems to be a key mechanism of change ${ }^{29}$ and is associated with higher quality of life ${ }^{30}$ and well-being. ${ }^{31}$

\section{Framework of the intervention}

The programme can be completed from home. Participants receive the self-help manual and work on one chapter per week for a period of 6 weeks. In addition, patients receive a phone-conversation with a qualified peer-counsellor per week in order to reflect on the specific chapter. Thereby, the 6-week intervention combines selfmanagement and peer-counselling. The peer counsellors receive a 2-day training beforehand, in which they work through the manual themselves and receive information on counselling techniques. In the first training for peercounsellors, we additionally asked them for feedback on all components of the programme and adapted the manuals accordingly. Changes involved mostly wording and examples given. To guide the telephone support, peer-counsellors further receive consulting guidelines that contain questions and additional information like example responses. They further receive supervision provided by a psychotherapist in order to receive support for their task.

\section{Content of the intervention}

The first chapter of the manual was designed specifically for each of the four diseases and contains information on the condition. Chapters 2-6 include generic modules with exercises based on ACT. See figure 2 for an overview of how the findings from prestudies were translated to design decisions.

\section{Information module}

The information module is the only disease-specific module. It contains a brief evidence-based description of the conditions derived from internet sources such as the National Institutes of Health (NIH) Genetic and Rare Diseases Information Center (https://rarediseases. info.nih.gov/). Moreover, during the focus groups, we collected questions of patients that they wished someone had explained to them in the past or in the present. We asked these questions to experts on the condition working at specialised outpatient clinics at the University Medical Center Hamburg-Eppendorf. Interviews with the experts were transcribed and given to the expert for proof. For each of the four conditions the first module consists of these general information and answers to questions raised by patients. Additionally, we provided information on how to identify a reliable source on the internet, based on WHO-recommendation.

\section{ACT modules}

Chapters 2-6 are generic and designed for all patients irrespective of condition. The second chapter addresses difficult emotions and how to deal with them. In the third chapter, patients are asked to differentiate between problems they can change and those they cannot and acceptance is introduced as a useful strategy for the latter. The forth chapter targets values by introducing the concept and engaging patients in extracting values of their own life, that is, what is really meaningful to them. The fifth chapter supports participants in formulating goals serving these values (value-based goals) and laying out a specific plan on how to address one chosen value-based goal. Finally, the last chapter contains reflecting on the whole program and writing a letter to patients' future selves. In addition to the manual, participants receive weekly telephone support by a peer counsellor of maximum $30 \mathrm{~min}$. During the phone calls, the recipients of the intervention are asked whether working on the current chapter worked and which difficulties arose. The recipients have the opportunity to ask questions regarding the content and process. Peer-counsellors are trained before the intervention starts. See figure 3 for an overview of framework and content of the intervention.

\section{DISCUSSION AND CONCLUSION \\ General discussion}

Patients with rare chronic diseases are exposed to psychosocial stress due to the course of their disease and difficult access to adequate care. ${ }^{12} 32$ The quantitative study we conducted in phase $1^{33}$ underlines the high psychological support need that has been postulated by patient organisations. ${ }^{6}$ In phase 2, we performed a qualitative focus group study specifying the support needs by asking what kind of support patients wish for and what has helped in the past. Some of the named aspects (eg, contact to peers, earlier diagnosis, more information) are in line with perceived problems, which patients described in other studies. ${ }^{2}$ Moreover, these aspects may reflect specific characteristics of the rare disease community that has been outlined by other authors as well. ${ }^{34}$ These findings stress the need for targeted psychosocial support for the rare disease community. The overall aim of the research project was the patient-centred development of a self-help service 
Phase 1: Survey for cross-disease quantitative and qualitative needs assessment ( $N=300$ participants; 81 rare diseases)

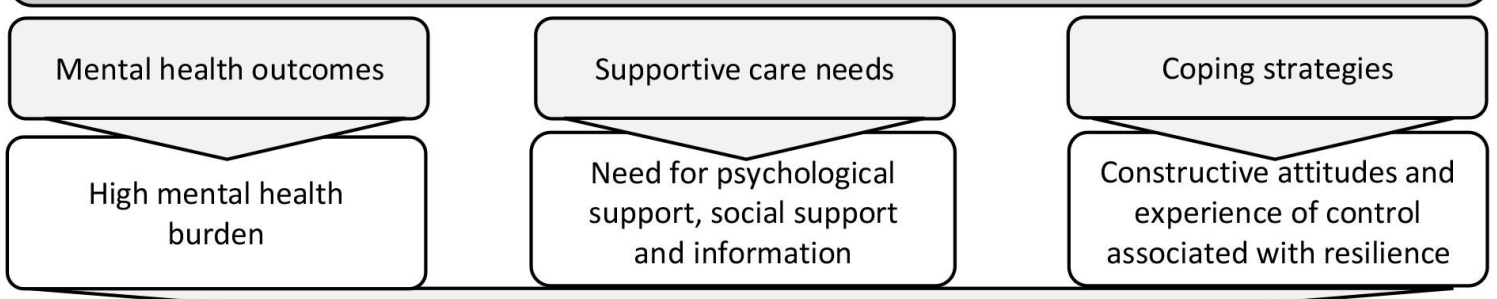

Phase 2: Focus groups for disease specific qualitative needs assessment

( $N=18$ participants; NF, PSC, PAH, MFS, disease homogeneous focus groups)

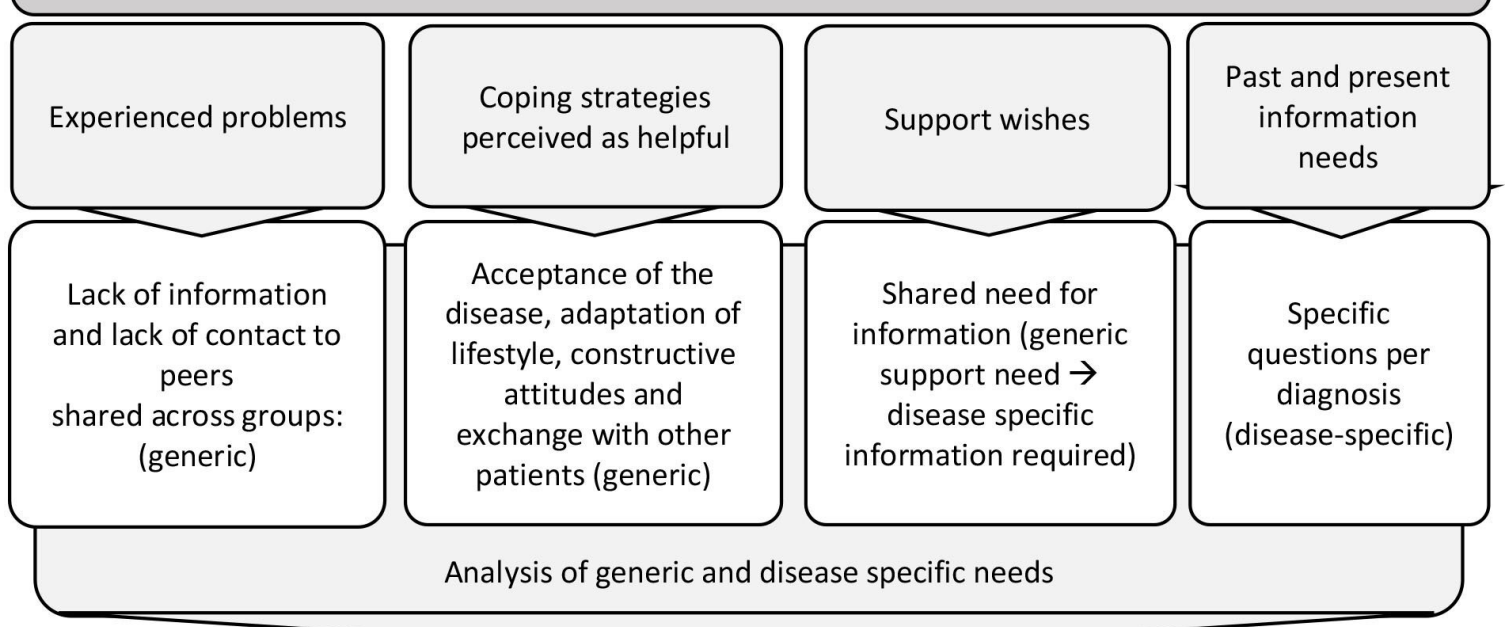

Phase 3: Development of a psychosocial intervention

\section{Literature search and consultation of experts}

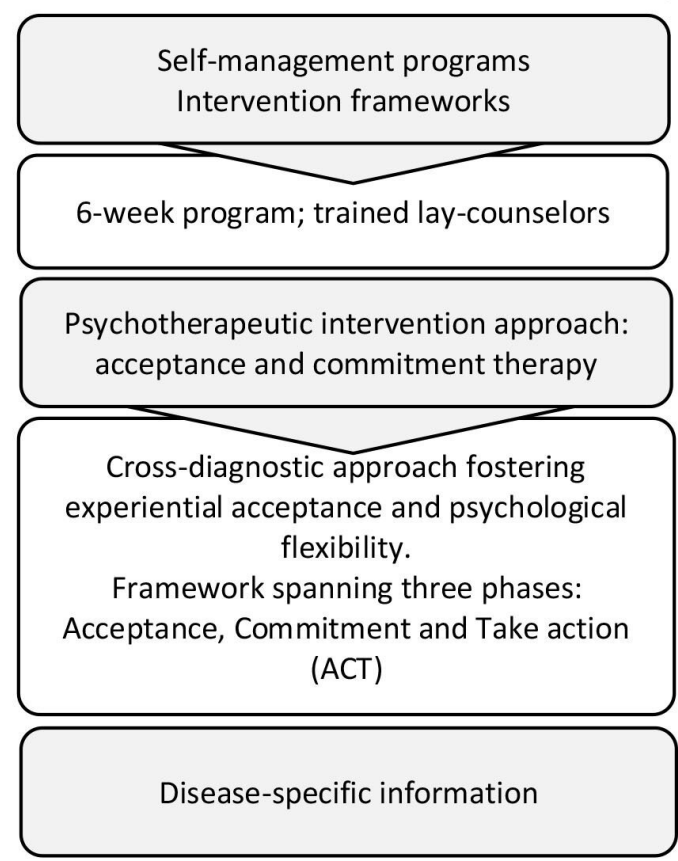

\section{Design}

Selection of exercises addressing the identified needs: dealing with difficult emotions, finding acceptance; adaptation of lifestyle and goals

Impact of disease on life and emotions Psychoeducation emotions

Encouraging experiential acceptance (ACT

"Tug of War with a Monster" metaphor)

Psychoeducation values and reflection Value-oriented specific goals

Writing manuals for recipients and counselors to introduce and link practices

Combining expert interviews and translated disease-specific information for information modules

Figure 2 Multistage development process. Overview of the results in the first two phases and the design process synthesing results and information from the literature. MFS, Marfan syndrome; NF, neurofibromatosis; PAH, pulmonary arterial hypertension; PSC, primary sclerosing cholangitis. 


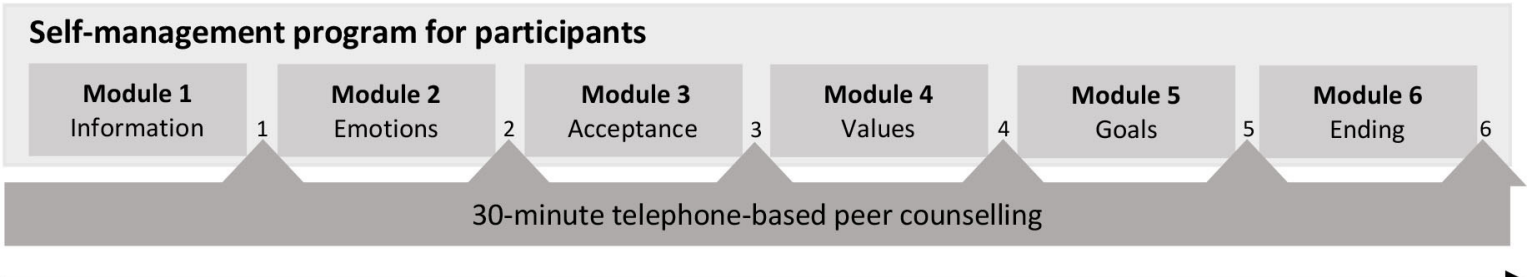

6-weeks

\section{Content of the self-management program}

Module 1: Information (disease-specific): General information about the diseases, interviews with experts, how to handle information from the internet; Exercise: impact of the disease on patients' lives

Module 2: Emotions (generic): Information about emotions and their function; Exercise: which difficult emotions do patients experience and which strategies to deal with them have (not) worked

Module 3: Acceptance (generic): ACT "Tug of War with a Monster" metaphor. Reflection on costs of fighting against things we cannot change; Exercise: which aspects of patients' situation can be changed and which not
Module 4: Values (generic): Information about values and their meaning for our lives; Exercise: what is important for patients and how well do they live in line with their values in different life areas

Module 5: Goals (generic): Information on how to set value based goals (SMART goals according to ACT); Exercise: deriving a concrete goal from a self-chosen value and detailed planning of realising this goal

Module 6: Ending (generic): Exercise: reflection of previous chapters (e.g. what was important to me? What do I want to implement in my life?) and letter to oneself in six months

\section{Consultation framework for peer counsellors}

2-days training before first consultation: working through the self-management program; information about counselling techniques; exercises and roleplays, e.g. on dealing with difficult situations during a counselling session Consultation guidelines: exemplary questions for each module e.g. what went well?/what was challenging?; additional information e.g. on values or on strategies to deal with difficult emotions

Supervision: mandatory after the first counselling session; on demand afterwards

Figure 3 Overview of the intervention. ACT, acceptance and commitment therapy.

tailored to the patient's needs. Synthesising past and the presented research findings, we developed an intervention that targets the needs of patients with rare diseases. In the development process, we build on the proven framework of self-management programmes. Based on the needs assessment, we designed a self-management manual that includes six modules on the following topics: Disease-specific information, dealing with negative emotions, accepting negative thoughts and emotions, values and value-based goals. We targeted the processes of acceptance and value-based action to facilitate following meaningful goals despite distress, which has been shown beneficial in the face of persisting symptoms like pain. ${ }^{27}$

The newly developed intervention targets the individual level by supporting patients in their coping with the condition and reflecting the attitude towards it to help foster constructive attitudes. Additionally, the intervention targets the patients' microsystem by introducing a peer counsellor as (temporary) part of the support system. Future research needs to investigate the effectiveness of this approach. While the intervention holds the potential to improve overall quality of life, other aspects of the patients' ecosystem remain unaltered, that is, given aspects of the healthcare system.

This project can be viewed as part of a growing body of research on the experiences of patients with rare chronic conditions and how these may be better addressed in care. ${ }^{34-38}$ Recurring themes are low quality of information available online ${ }^{38}$ and need for contact to patients with the same condition. ${ }^{35}$ Recent research indicates that companionship and emotional support (rather than informational support) are beneficial for patients with rare diseases. ${ }^{36}$ Based on their findings, the authors recommend that intervention research should promote more companionship and emotional support to improve the life satisfaction of patients affected by rare diseases. ${ }^{36}$ Peer support in a structured programme is one way to implement this recommendation. A major strength of this intervention is the patient-centred development and direct deduction from the self-reported wishes of the target group. The approach allows addressing needs of a hard to reach group of patients. Moreover, most parts of the intervention are generic and thus enable an implementation for different rare diseases, which is particularly beneficial in view of the high number of different 
rare diseases. Peer counsellors can help address the wanted exchange with other patients affected by the same rare condition. They further contribute their individual disease specific knowledge. Along those lines, a support conference for a single rare disease, Moebius syndrome, increased perceived knowledge shown in a predesign-postdesign. ${ }^{39}$ Additionally, it may be an economically efficient approach in the face of finite healthcare resources. While the intervention may help improve overall quality of life by fostering constructive coping, it cannot replace psychotherapy or address specific psychiatric conditions. Some authors showed that counselling benefits the lay counsellors, ${ }^{40}$ however, it may also be challenging for the providers of the intervention and could evoke experiences of helplessness in the face of these challenges. Another limitation is that the development targets both shared needs as well as diseasespecific information, limiting it's generalisability to other rare diseases. Finally, the literature search we conducted in phase 3 was not conducted as systematic review and therefore does not present a comprehensive picture of the corresponding literature.

\section{Conclusion}

In chronic conditions, self-management approaches have the potential to strengthen the affected individuals as they become their own primary caregivers. ${ }^{4}$ The newly developed intervention adapts the proven framework of self-management programmes in chronic conditions to the care needs of patients with rare chronic conditions. While we present the development process of the intervention, we do not address whether the intervention is accepted, feasible and efficacious. This was subject of a randomised control trial and will be reported separately.

\section{Practice implications}

Combining self-management, peer-counselling and ACT may help living with a rare condition. Future research needs to investigate acceptance and effectiveness of the novel intervention prior to implementing it into general care.

Acknowledgements This study is part of the project 'Patients for patients: qualified peer counselling and self-management for patients with rare chronic diseases', a research project aiming to develop and evaluate a self-management program for patients with different rare chronic diseases. We would like to thank our partners ACHSE e.V. and the Martin Zeitz Center for Rare Diseases at the University Medical Center Hamburg-Eppendorf for their cooperation and support. We thank Lance McCracken for allowing us to observe his stationary ACT treatment and the exchange. We are grateful to all patients who supported us by participating in the study. We would further like to thank Anne Marie-Waßmuth (AW), Laura Holz (LH) and Johanna Dreyer (JD) for supporting data analysis. We thank Lukas Feuer for assisting in literature search and formatting of study material.

Contributors All authors contributed to conception, design, acquisition, analysis or interpretation of this study. Further, all authors contributed to writing or editing the draft of the manuscript and approved to the final version. Finally, all authors agree to be accountable for all aspects of the work. MKD: conceptualisation, methodology, formal analysis, investigation, data curation, writing-original draft, supervision, project administration, funding acquisition. NU: methodology, formal analysis, investigation, data curation, writing-original draft method and results of phase 2, writing-review and editing. BL: conceptualisation, resources, writing-review and editing, supervision, project administration, funding acquisition.

Funding This work was supported by Robert Bosch Stiftung (32.5.A401.0004.0).
Disclaimer The foundation provided funding in the form of salaries for authors (NU) and research materials but did not play a role in the study design, data collection and analysis, decision to publish or preparation of the manuscript.

\section{Competing interests None declared.}

Patient consent for publication Not required.

Ethics approval Ethics approval was given by the independent ethics committee of the Hamburg Medical Chamber (Ärztekammer Hamburg) on 2 February 2016 (reference number PV5088).

Provenance and peer review Not commissioned; externally peer reviewed.

Data availability statement Data are available on reasonable request. Data cannot be made publicly available due to ethical reasons. In the area of rare diseases, information about the diagnosis in combination with personal information may compromise anonymity and confidentiality of the participants. The independent ethics committee of the Hamburg Medical Chamber assessed our research project beforehand. The ethics vote allows sharing data with eligible researchers but we do not have approval to share the data publicly. Researchers interested in getting access to the data should feel free to contact the principal investigator $(B L-b$. loewe@uke.de).The ethics committee can be contacted at: ethik@aekhh.de.

Supplemental material This content has been supplied by the author(s). It has not been vetted by BMJ Publishing Group Limited (BMJ) and may not have been peer-reviewed. Any opinions or recommendations discussed are solely those of the author(s) and are not endorsed by BMJ. BMJ disclaims all liability and responsibility arising from any reliance placed on the content. Where the content includes any translated material, BMJ does not warrant the accuracy and reliability of the translations (including but not limited to local regulations, clinical guidelines, terminology, drug names and drug dosages), and is not responsible for any error and/or omissions arising from translation and adaptation or otherwise.

Open access This is an open access article distributed in accordance with the Creative Commons Attribution Non Commercial (CC BY-NC 4.0) license, which permits others to distribute, remix, adapt, build upon this work non-commercially, and license their derivative works on different terms, provided the original work is properly cited, appropriate credit is given, any changes made indicated, and the use is non-commercial. See: http://creativecommons.org/licenses/by-nc/4.0/.

\section{ORCID iDs}

Miriam K Depping http://orcid.org/0000-0003-3205-6060

Natalie Uhlenbusch http://orcid.org/0000-0002-4031-9839

\section{REFERENCES}

1 European Organisation for Rare Diseases. What is a rare disease? 2010.

2 von der Lippe C, Diesen PS, Feragen KB. Living with a rare disorder: a systematic review of the qualitative literature. Mol Genet Genomic Med 2017;5:758-73.

3 Stanton AL, Revenson TA, Tennen H. Health psychology: psychological adjustment to chronic disease. Annu Rev Psychol 2007;58:565-92.

4 Bodenheimer T, Lorig K, Holman $\mathrm{H}$, et al. Patient self-management of chronic disease in primary care. JAMA 2002;288:2469-75.

5 Clarke DM, Currie KC. Depression, anxiety and their relationship with chronic diseases: a review of the epidemiology, risk and treatment evidence. Med J Aust 2009;190:54-60.

6 European Organisation for Rare Diseases. Rare diseases: understanding this public health priority. France: Paris: European Organisation for Rare Diseases, 2005.

7 Eidt D, Frank M, Reimann A. Maßnahmen Zur Verbesserung Der gesundheitlichen situation von Menschen MIT seltenen Erkrankungen in Deutschland. Bundesgesundheitsblatt Gesundheitsforschung Gesundheitsschutz 2009.

8 Cohen JS, Biesecker BB. Quality of life in rare genetic conditions: a systematic review of the literature. Am J Med Genet $A$ 2010;152A:1136-56.

9 Kroenke K, Spitzer RL. The PHQ-9: a new depression diagnostic and severity measure. Psychiatr Ann 2002;32:509-15.

10 Löwe B, Kroenke K, Herzog W, et al. Measuring depression outcome with a brief self-report instrument: sensitivity to change of the patient health questionnaire (PHQ-9). J Affect Disord 2004;81:61-6.

11 Martin A, Rief W, Klaiberg A, et al. Validity of the brief patient health questionnaire mood scale (PHQ-9) in the general population. Gen Hosp Psychiatry 2006;28:71-7.

12 Spitzer RL, Kroenke K, Williams JBW, et al. A brief measure for assessing generalized anxiety disorder: the GAD-7. Arch Intern Med 2006;166:1092-7. 
13 Löwe B, Decker O, Müller S, et al. Validation and standardization of the generalized anxiety disorder screener (GAD-7) in the general population. Med Care 2008;46:266-74.

14 Uhlenbusch N, Löwe B, Härter M, et al. Depression and anxiety in patients with different rare chronic diseases: a cross-sectional study. PLoS One 2019;14:e0211343.

15 Depping MK, Uhlenbusch N, von Kodolitsch Y, et al. Supportive care needs of patients with rare chronic diseases: multi-method, crosssectional study. Orphanet J Rare Dis 2021;16.

16 Stewart DE, Yuen T. A systematic review of resilience in the physically ill. Psychosomatics 2011;52:199-209.

17 Mayring P, Fenzl T. Qualitative Inhaltsanalyse [Qualitative content analysis]. In: Baur N, Blasius J, eds. Handbuch Methoden der empirischen Sozialforschung [Handbook methods of empirical social research]. Springer Wiesbaden, 2014: 543-56.

18 Uhlenbusch N, Löwe B, Depping MK. Perceived burden in dealing with different rare diseases: a qualitative focus group study.

19 Hayes SC, Luoma JB, Bond FW, et al. Acceptance and commitment therapy: model, processes and outcomes. Behav Res Ther 2006;44:1-25.

20 Hayes S, Strosahl K, Wilson K. Acceptance and commitment therapy: an experiential approach to behavior change. New York: Guilford Press, 1999

21 Graham CD, Gouick J, Krahé C, et al. A systematic review of the use of acceptance and commitment therapy (ACT) in chronic disease and long-term conditions. Clin Psychol Rev 2016;46:46-58.

22 Barlow J, Wright C, Sheasby J, et al. Self-management approaches for people with chronic conditions: a review. Patient Educ Couns 2002:48:177-87.

23 Lorig KR, Sobel DS, Ritter PL, et al. Effect of a self-management program on patients with chronic disease. Eff Clin Pract 2001;4:256-62.

24 Lorig KR, Sobel DS, Stewart AL, et al. Evidence suggesting that a chronic disease self-management program can improve health status while reducing hospitalization: a randomized trial. Med Care 1999;37:5-14.

25 Sokol R, Fisher E. Peer support for the hardly reached: a systematic review. Am J Public Health 2016;106:e1-8.

26 Brassington L, Ferreira NB, Yates S, et al. Better living with illness: a transdiagnostic acceptance and commitment therapy group intervention for chronic physical illness. J Contextual Behav Sci 2016;5:208-14.
27 Vowles KE, McCracken LM. Acceptance and values-based action in chronic pain: a study of treatment effectiveness and process. $J$ Consult Clin Psychol 2008;76:397-407.

28 Veehof MM, Oskam M-J, Schreurs KMG, et al. Acceptance-based interventions for the treatment of chronic pain: a systematic review and meta-analysis. Pain 2011;152:533-42.

29 Cederberg JT, Cernvall M, Dahl J, et al. Acceptance as a mediator for change in acceptance and commitment therapy for persons with chronic pain? Int J Behav Med 2016;23:21-9.

30 Poppe C, Crombez G, Hanoulle I, et al. Improving quality of life in patients with chronic kidney disease: influence of acceptance and personality. Nephrol Dialysis Transplant 2013;28:116-21.

31 Van Damme S, Crombez G, Van Houdenhove B, et al. Well-being in patients with chronic fatigue syndrome: the role of acceptance. J Psychosom Res 2006;61:595-9.

32 European Organization for Rare Diseases. The voice of rare disease patients: experiences and expectations of over 3,000 patients on rare disease patient registries in Europe. European Organization for Rare Diseases, 2013.

33 Depping MK, Uhlenbusch N, von Kodolitsch Y, et al. Supportive care needs of patients with rare chronic diseases: multi-method, crosssectional study. Orphanet J Rare Dis 2021;16:44

34 Bryson B, Bogart K, Atwood M, et al. Navigating the unknown: a content analysis of the unique challenges faced by adults with rare diseases. J Health Psychol 2019:135910531982815.

35 Bogart KR, Irvin VL. Health-related quality of life among adults with diverse rare disorders. Orphanet $J$ Rare Dis 2017;12:1-9.

36 Bryson BA, Bogart KR. Social support, stress, and life satisfaction among adults with rare diseases. Health Psychol 2020;39:912-20.

37 Litzkendorf S, Babac A, Rosenfeldt D. Information needs of people with rare diseases-what information do patients and their relatives require? J Rare Dis Diagnosis Therapy 2016.

38 Pauer F, Litzkendorf S, Göbel J, et al. Rare diseases on the internet: an assessment of the quality of online information. $J$ Med Internet Res 2017;19:e23

39 Bogart KR, Frandrup E, Locke T, et al. "Rare place where I feel normal": perceptions of a social support conference among parents of and people with Moebius syndrome. Res Dev Disabil 2017;64:143-51.

40 MacLellan J, Surey J, Abubakar I, et al. Peer support workers in health: a qualitative metasynthesis of their experiences. PLoS One 2015;10:e0141122. 